\title{
Machinery that guides immunity
}

The peptide-loading complex is key to the initiation of an immune response that raises killer $\mathrm{T}$ cells in vertebrates. Its structure has now been determined, and might provide information that improves immune protection. SEE LETTER P.525

\section{HIDDE PLOEGH}

$\mathrm{T}$ he vertebrate immune system uses membrane glycoproteins as the guidance system for its 'missiles'. Formed by cellular machinery called the major histocompatibility complex (MHC), these glycoproteins present protein fragments (peptides) derived from viral and bacterial invaders to immune cells called killer T lymphocytes. These cells, along with other cell types, can then engage with the invader and mount an immune response to protect the host. On page 525, Blees et al. ${ }^{1}$ report a structure of the human peptide-loading complex (PLC), the molecular machine that charges MHC products with their peptide cargo, and which is therefore essential for the initiation of the killer T cell's response.

There are two flavours of MHC product, known as MHC class I and class II molecules. MHC class I molecules are displayed at the cell surface, but they derive their peptide cargo mostly from proteins in the cytosol. Specialized apparatus is needed to ferry peptides from the cytosol into a cellular organelle called the endoplasmic reticulum (ER), where they are loaded by the PLC onto MHC class I molecules. Once loading is complete, the peptide-occupied MHC molecule leaves the ER and travels to the cell surface ${ }^{2}$. The process is not perfect: a small fraction of 'empty' MHC class I molecules can escape from the PLC and still be displayed at the cell surface as peptidereceptive molecules, a trait exploited by immunologists when testing peptides for their ability to be presented by MHC products ${ }^{3}$.

The PLC consists of two subunits of the MHC class I molecule, together with a set of protein accessories ${ }^{2}$. These include: TAP1 and TAP2, which form a heterodimer known simply as TAP, and which move peptides of preferred length and sequence into the ER; tapasin, which interacts with TAP; and ERp57, which is needed for tapasin to function in the assembly of MHC class I products. Calreticulin, a protein involved in the sensing of nascent glycoproteins ${ }^{4}$, also forms part of the PLC, where it recruits MHC class I molecules to the complex.

The importance of the PLC is illustrated by the fact that evolution has endowed a variety

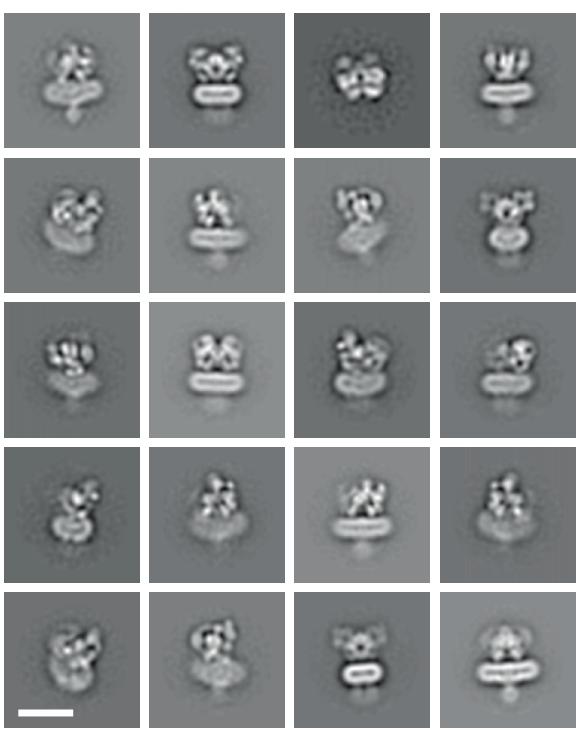

Figure 1 | The human peptide-loading complex (PLC). Blees et al. ${ }^{1}$ report the structure of the human PLC - cellular machinery that performs a crucial step in the initiation of an immune response. The authors produced a gallery of images (some of which are pictured) of single particles of the purified PLC using cryo-electron microscopy, and analysed these to obtain an average structure. Scale bar, 10 nanometres.

of viruses with the means of disabling it. For example, herpes simplex virus 1 produces the ICP47 protein, which binds tightly to TAP and blocks peptide translocation ${ }^{5}$. Blees et al. used a tagged version of ICP47 as a handle with which to pull PLC out of extracts from no less than 250 litres of cultured lymphoma cells. The authors then used a technique called singleparticle cryo-electron microscopy (cryo-EM) to produce a gallery of images of the purified PLC (Fig. 1), from which the structure of the complex emerged.

To work out the full structure, Blees and colleagues constrained the possible range of the distances between protein subunits by analysing fragments of the PLC that could be connected by chemical crosslinks. They also used information from previously obtained structures of isolated PLC components, including: X-ray structures of the MHC class I molecule; the X-ray structure ${ }^{6}$ of the complex formed by tapasin and ERp57; a structure ${ }^{7}$ of part of calreticulin, obtained by nuclear magnetic resonance spectroscopy; and the recently published cryo-EM structure of the ICP47-TAP1-TAP2 complex ${ }^{8}$. The authors' cryo-EM data provided a $3 \mathrm{D}$ outline of the PLC, into which the previously obtained structures could be fitted to obtain a detailed PLC structure.

Not all the components of the PLC could be visualized with equal resolution in the cryoEM structure, suggesting that dynamic alterations occur as the PLC performs its functions. This is to be expected for a protein complex that not only responds to the occupancy of the TAP peptide transporters ${ }^{9}$, but also distinguishes between conformational states of class I MHC products.

Blees and co-workers found that either one or both of the editing complexes - the modules of the PLC that allow peptides to be loaded onto MHC class I molecules are occupied by an MHC class I molecule in complex with calreticulin. This suggests that one of the two editing complexes can adopt an 'incomplete' state that lacks a class I molecule (either with or without calreticulin), yielding an asymmetric PLC. The overall cryo-EM structure suggests that peptides arriving in the ER through TAP can escape the PLC to be processed by ER-resident peptidase enzymes; the processed peptides can then bind to MHC class I molecules. The asymmetric arrangement mentioned above implies the existence of a toggle mechanism, in which the vacation of a PLC site by a peptide-loaded MHC product allows insertion of the next empty MHC molecule waiting in line.

The new PLC structure does not tell the complete story of how MHC class I molecules acquire peptides. Two papers ${ }^{10,11}$ have recently documented a structure of MHC class I molecules in complex with a protein called TAPbinding protein-related (TAPBPR). TAPBPR is distantly related to tapasin (with which it shares $22 \%$ of its amino-acid sequence), and also forms a transient complex with empty MHC class I molecules. However, TAPBPR and tapasin bind preferentially to different allelic variants of those molecules (which are produced by variants of the gene that encodes them). TAPBPR's job is to scan the quality of newly formed peptide-MHC class I complexes 
by interacting with the structural elements that anchor the peptide's carboxy terminus in a pocket of the MHC class I molecule's peptidebinding groove.

The structure ${ }^{10,11}$ of MHC class I molecules in complex with TAPBPR shows that the latter molecule does not detectably interact with ERp57, and that its binding site on the MHC class I molecule overlaps with the tapasin binding site. A structural feature of TAPBPR dubbed the scoop loop ${ }^{10}$ helps to displace peptides that have modest affinity for the MHC molecule by competing for interaction with amino-acid residues in the groove that would otherwise stabilize interactions between the MHC molecule and the peptide.

Unlike tapasin, TAPBPR is found in locations other than the ER, and might even act downstream of the PLC as the final arbiter of the overall quality of newly formed MHC class I-peptide complexes. In the ER, TAPBPR associates with an enzyme called UDP glucose glycoprotein glycosyltransferase, which, in conjunction with other ER-resident enzymes, acts as a timer for the engagement and release of MHC class I molecules by the calnexin-calreticulin cycle (which controls glycoprotein maturation in the ER; ref. 4). Possible discrepancies between the way in which the calnexin-calreticulin cycle in the ER controls folding and assembly of MHC class I molecules, and TAPBPR-imposed quality control elsewhere in the cell, can now be addressed in greater detail.

Why does it help to better understand peptide presentation by MHC class I molecules? The visualization of the PLC in almost atomic detail might yield actionable information, for example by suggesting ways in which viral disablers of peptide presentation can be intercepted ${ }^{12}$, or strategies to improve the presentation of peptides that are recognized on cancer cells. Tumour immunologists are increasingly turning their eye to neoantigens (variants of normal peptides that form only in cancer cells, and which can elicit an immune response when in complex with $\mathrm{MHC}$ products) as targets for immunotherapy ${ }^{13}$. So, the more detailed our understanding of how MHC products are put together, the better will be our predictions of how to manipulate immune processes and affect outcomes.

Hidde Ploegh is in the Program of Cellular and Molecular Medicine, Boston Children's Hospital, Boston, Massachusetts 02115, USA. e-mail:hidde.ploegh@childrens.harvard.edu

1. Blees, A. et al. Nature 551, 525-528 (2017).

2. Rock, K. L., Reits, E. \& Neefjes, J. Trends Immunol. 37, 724-737 (2016).

3. Ljunggren, H.-G. et al. Nature $\mathbf{3 4 6}$, $\mathbf{4 7 6}-\mathbf{4 8 0}$ (1990).

4. Ellgaard, L., McCaul, N., Chatsisvili, A. \& Braakman, I. Traffic 17, 615-638 (2016).

5. Hill, A. et al. Nature 375, 411-415 (1995).

6. Dong, G., Wearsch, P. A., Peaper, D. R. \& Cresswell, P. \& Reinisch, K. M. Immunity 30, 21-32 (2009).
7. Frickel, E.-M. et al. Proc. Natl Acad. Sci. USA 99, 1954-1959 (2002).

8. Oldham, M. L et al. Nature 529, 537-540 (2016).

9. Reits, E. A. J., Vos, J. C., Grommé, M \& Neefjes, J. Nature 404, 774-778 (2000).

10.Thomas, C. \& Tampé, R. Science http://dx.doi. org/10.1126/science.aao6001 (2017).
11.Jiang, J. et al. Science http://dx.doi.org/10.1126/ science.aao5154 (2017)

12.Schuren, A. B. C., Costa, A. I. \& Wiertz, E. J. H. J. Curr. Opin. Immunol. 40, 43-50 (2016).

13.Yarchoan, M., Johnson. B. A. III, Lutz, E. R. Laheru, D. A. \& Jaffee, E. M. Nature Rev. Cancer 17, 209-222 (2017).

\section{ATMOSPHERIC SCIENCE}

\section{Thunderous nuclear reactions}

The discovery that thunderstorms can trigger nuclear reactions provides insight into the physics of atmospheric electricity and unveils a previously unknown natural source of radioactive isotopes on Earth. SEE LETTER P. 481

\section{LEONID BABICH}

1 Thunderstorms are some of nature's most spectacular phenomena. Almost a century ago, it was suggested that the strong electric fields in thunderclouds could accelerate electrons in the atmosphere and induce nuclear reactions ${ }^{1}$. However, these processes have been difficult to confirm experimentally. On page 481, Enoto et al. ${ }^{2}$ report the first conclusive observational evidence for thunderstorm-produced nuclear reactions - with implications for our understanding of Earth's atmosphere and isotopic composition.

The idea that thunderstorms can trigger nuclear reactions was proposed ${ }^{1}$ by the Scottish physicist and meteorologist Charles Wilson in 1925. However, the state of physics at the time meant that Wilson could not fully substantiate his idea. For instance, it is now known that neutrons are among the possible products of nuclear reactions and therefore that detecting these particles from a thunderstorm would provide evidence for Wilson's proposal. But neutrons were not discovered ${ }^{3}$ until 1932.

Thunderstorms occur in the dense lowermost layers of the atmosphere. Electrons in these layers undergo frequent collisions with air molecules and are therefore subject to a strong drag force. Wilson's proposal requires electrons that have sufficiently high initial energies to overcome this force. It is now known that cosmic rays irradiate the atmosphere and produce such electrons, which multiply in thunderclouds to form an avalanche of highenergy electrons ${ }^{4}$. However, in the mid-1920s, cosmic rays were extremely mysterious and thought to be of terrestrial origin ${ }^{5}$.

The first claimed detection of neutrons from a thunderstorm was reported ${ }^{6}$ in 1985 . These

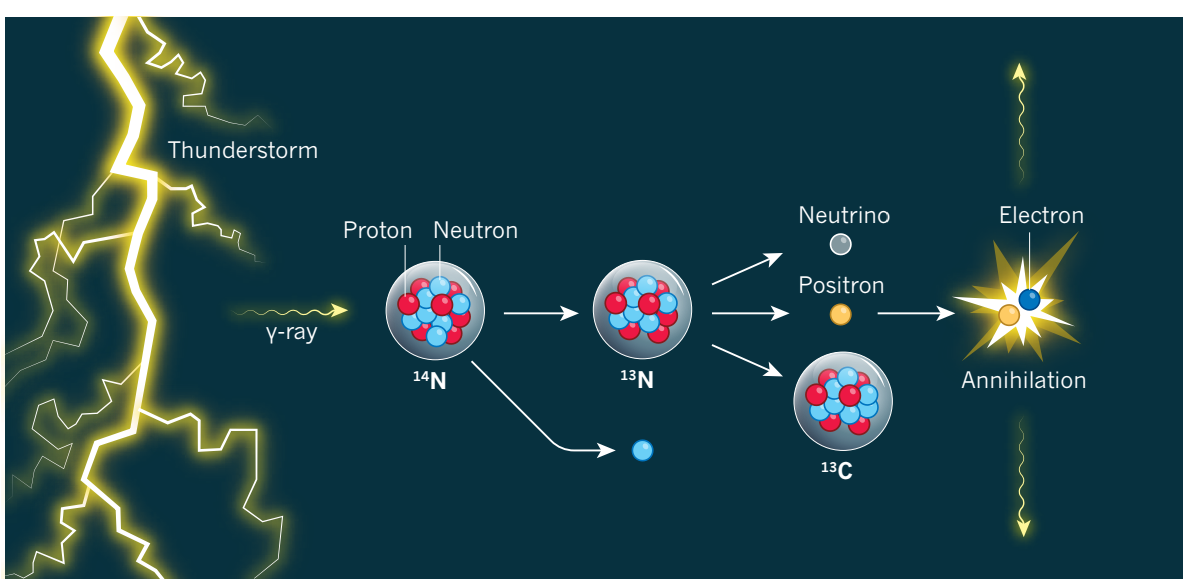

Figure 1 | Nuclear reactions triggered by a thunderstorm. Enoto et al. ${ }^{2}$ report conclusive evidence that thunderstorms can induce nuclear reactions in the atmosphere. For example, the authors find that a thunderstorm can generate a high-energy $\gamma$-ray that knocks a neutron out of a nitrogen-14 nucleus, creating an unstable nitrogen-13 isotope. The isotope decays into a neutrino, a positron (the antiparticle of the electron) and a stable carbon-13 nucleus. Finally, the positron annihilates with an electron of an atmospheric molecule to produce a pair of $\gamma$-rays, each of which has a characteristic energy (0.511 megaelectronvolts). 